\title{
SHARP ESTIMATES FOR GREEN POTENTIALS ON NON-SMOOTH DOMAINS
}

\author{
Svitlana Mayboroda and Marius Mitrea
}

\section{Introduction}

Given an open, bounded, connected domain $\Omega \subset \mathbb{R}^{n}$, let $\mathbf{G}_{D}, \mathbf{G}_{N}$ be the solution operators for the Poisson equation for the Laplacian in $\Omega$ with homogeneous Dirichlet and Neumann boundary conditions, respectively. The aim of this note is to describe the sharp ranges of indices for which $\mathbf{G}_{D}, \mathbf{G}_{N}$ are smoothing operators of order two, on Besov and Triebel-Lizorkin scales in arbitrary Lipschitz domains. This builds on the work of many people who have dealt with homogeneous/inhomogenous problems for the Laplacian with Dirichlet/Neumann boundary conditions in Lipschitz domains; an excellent account can be found in [23]. Earlier results emphasized homogeneous problems with boundary data exhibiting an integer amount of smoothness. Such estimates underpin the entire work here and play the role of end-point/limiting cases in our theory.

The main theorems we prove extend the work of D. Jerison and C. Kenig [20], whose methods and results are largely restricted to the case $p \geq 1$, and answer the open problem \# 3.2.21 on p. 121 in C. Kenig's book [23] in the most complete fashion. When specialized to Hardy spaces (viewed as a subclass of Triebel-Lizorkin scale), our results provide a solution of a (strengthened form of a) conjecture made by D.-C. Chang, S. Krantz and E. Stein regarding the regularity of the Green potentials on Hardy spaces in Lipschitz domains. Cf. p. 130 of [5] where the authors write: "For some applications it would be desirable to find minimal smoothness conditions on $\partial \Omega$ in order for our analysis of the Dirichlet and Neumann problems to remain valid. We do not know whether $C^{1+\varepsilon}$ boundary is sufficient in order to obtain [Hardy space] estimates for the Dirichlet problem when $p$ is near $1 .[\ldots]$

The literature for the Dirichlet and Neumann problems for domains with Lipschitz boundaries (see [23]) teaches us that when the boundary is only Lipschitz then one can expect favorable behavior for a restricted range of $p$. It would be of

Received March 18, 2004.

2000 Math Subject Classification. Primary: 35J25, 42B20, 46E35. Secondary 35J05, 31B10, $42 \mathrm{~B} 30$.

Key words: Besov and Triebel-Lizorkin spaces, Lipschitz domains, traces, Green potentials, elliptic PDE..

The work of M. Mitrea was supported in part by grants from NSF and the UM Office of Research. 
interest to explore similar phenomena vis à vis to the Hardy spaces introduced here."

The corollaries of our main results include new proofs and various extensions of: (i) Hardy space estimates for Green potentials in convex domains due to V. Adolfsson, B. Dahlberg, S. Fromm, D. Jerison, G. Verchota and T. Wolff [1], [2], [14]. and (ii) the $L^{p}-L^{q}$ estimates for the gradients of Green potentials in Lipschitz domains, due to B. Dahlberg [7].

As a key prerequisite, in $\S 2$ we include a discussion of sharp results for tangential and normal traces of Besov and Triebel-Lizorkin scales on Lipschitz domains. More references and bibliographical comments are included with each section. Full details will appear elsewhere.

We now briefly elaborate on notation and terminology. An open, connected set $\Omega \subset \mathbb{R}^{n}$ is called a Lipschitz domain if its boundary can be locally described by means of Lipschitz graphs (in appropriate systems of coordinates); see, e.g., [28] for a more detailed discussion. Given $\Omega \subset \mathbb{R}^{n}$ Lipschitz and $0<p, q \leq \infty$, $\alpha \in \mathbb{R}$, we set

$$
\begin{aligned}
& B_{\alpha}^{p, q}(\Omega):=\left\{u \in \mathcal{D}^{\prime}(\Omega): \exists v \in B_{\alpha}^{p, q}\left(\mathbb{R}^{n}\right) \text { with }\left.v\right|_{\Omega}=u\right\}, \\
& B_{\alpha, 0}^{p, q}(\Omega):=\left\{u \in B_{\alpha}^{p, q}\left(\mathbb{R}^{n}\right) \text { with } \operatorname{supp} u \subseteq \bar{\Omega}\right\},
\end{aligned}
$$

with similar definitions for $F_{\alpha}^{p, q}(\Omega)$ and $F_{\alpha, 0}^{p, q}(\Omega)$. Finally, $B_{s}^{p, q}(\partial \Omega)$ stands for the Besov class on the Lipschitz manifold $\partial \Omega$, obtained by transporting (via a partition of unity and pull-back) the standard scale $B_{s}^{p, q}\left(\mathbb{R}^{n-1}\right)$. General references for these smoothness spaces are [13], [28], [31].

\section{Tangential and normal traces}

2.1. Traces of Besov and Triebel-Lizorkin spaces. The issue of traces is central in the context of boundary value problems. To state our main result in this regard, let $(a)_{+}:=\max \{a, 0\}$.

Theorem 2.1. Let $\Omega$ be a Lipschitz domain in $\mathbb{R}^{n}$ and assume that $(n-1)\left(\frac{1}{p}-\right.$ $1)_{+}<s<1$. Then the restriction to the boundary extends to a linear, bounded operator

$$
\operatorname{Tr}: B_{s+\frac{1}{p}}^{p, q}(\Omega) \longrightarrow B_{s}^{p, q}(\partial \Omega) \quad \text { for } 0<q \leq \infty .
$$

Moreover, for this range of indices, $\operatorname{Tr}$ is onto and has a bounded right inverse

$$
\operatorname{Ex}: B_{s}^{p, q}(\partial \Omega) \longrightarrow B_{s+\frac{1}{p}}^{p, q}(\Omega) .
$$

Similar considerations hold for $\operatorname{Tr}: F_{s+\frac{1}{p}}^{p, q}(\Omega) \rightarrow B_{s}^{p, p}(\partial \Omega)$ with $s, p$ as before and for suitable values of $q$.

Results of this type have a long history. Some of the forerunners can be found in the books by Nikol'skij [26], Peetre [27] and Triebel [30], where the case $\Omega=\mathbb{R}_{+}^{n}, p \geq 1$ has been treated. Cf. also [29] for $p=1$ and the discussion in [28]. Jawerth was the first to consider the case $p<1$ (still for the upper-half space) in [18]; cf. also [11], [12], [13] in this regard. Adaptations to smooth 
domains are found in [10] and [31]. Dealing with non-smooth domains raises a whole new set of challenges and the case $p \geq 1$ has been dealt with in [22] for a class of domains including those having a Lipschitz boundary; see also the discussion in [20].

Here we extend this work by dealing with the optimal range of indices in the context of arbitrary Lipschitz domains. In [22] the authors have constructed an extension operator by decomposing $\mathbb{R}^{n} \backslash \partial \Omega$ in Whitney cubes and gluing local averages over such cubes via a suitable partition of unity, but the argument breaks down when dealing with Besov and Triebel-Lizorkin spaces which are not locally convex. By way of comparison, our approach employs singular integral operators (an idea which seems to be new) and - as long as $0<s<1$, which is natural given that $\partial \Omega$ is only Lipschitz - works for the same range of indices as in the case of the upper-half space.

As far as the class of Lipschitz domains is concerned, Theorem 2.1 satisfactorily settles the issues under discussion. It also further reinforces the philosophy that the Lipschitz domains make up the most general class of domains where a rich function theory can be developed, comparable in power and scope with that associated with the upper-half space.

2.2. The normal derivative. Having developed a satisfactory trace theory in Lipschitz domains, extending the definition of the normal derivative, i.e., $\partial_{\nu} u=\nu \cdot \operatorname{Tr}[\nabla u]$, from functions in $C^{1}(\bar{\Omega})$ to functions belonging to Besov and Triebel-Lizorkin scales is a straightforward step if the smoothness index is large; however, this is considerably more delicate if $u$ is not regular enough so that $\nabla u$ has a trace in the classical sense. Here we describe a procedure which takes into account not only the smoothness of the function $u$ itself but the smoothness of the Laplacian of $u$ as well. In fact, our definition of the normal derivative strongly depends on the choice of an extension $f$ of $\Delta u$ to a distribution in $\mathbb{R}^{n}$ supported in $\bar{\Omega}$. The notation, $\partial_{\nu}^{f} u$, is chosen to reflect this peculiarity.

When $1<p, q<\infty, 0<s<1, u \in B_{s+\frac{1}{p}}^{p, q}(\Omega)$ and $f \in B_{s+\frac{1}{p}-2,0}^{p, q}(\Omega)$ are such that $\Delta u=\left.f\right|_{\Omega}$, then one can define $\partial_{\nu}^{f} u \in B_{s-1}^{p, q}(\partial \Omega)=\left(B_{1-s}^{p^{\prime}, q^{\prime}}(\partial \Omega)\right)^{*}$, $1 / p+1 / p^{\prime}=1,1 / q+1 / q^{\prime}=1$, by

$$
\left\langle\partial_{\nu}^{f} u, \psi\right\rangle:=\langle f, \operatorname{Ex}(\psi)\rangle+\langle\nabla u, \nabla \operatorname{Ex}(\psi)\rangle, \quad \forall \psi \in B_{1-s}^{p^{\prime}, q^{\prime}}(\partial \Omega),
$$

where Ex is the extension operator in (2.2). Note that all the pairings in the right-hand side of (2.3) are well-defined. This is essentially the point of view adopted in [9] but its applicability is limited to the range $1<p, q \leq \infty$, as $B_{s-1}^{p, q}(\partial \Omega)$ fails to be a dual space if $\min \{p, q\} \leq 1$. We nonetheless have:

Theorem 2.2. Let $\Omega$ be a bounded Lipschitz domain in $\mathbb{R}^{n}$ and assume that $(n-1)\left(\frac{1}{p}-1\right)_{+}<s<1,0<q \leq \infty$. Then one can define a concept of normal 
derivative such that

$$
\left\{(u, f) \in B_{s+\frac{1}{p}}^{p, q}(\Omega) \oplus B_{s+\frac{1}{p}-2,0}^{p, q}(\Omega): \Delta u=\left.f\right|_{\Omega}\right\} \ni(u, f) \mapsto \partial_{\nu}^{f} u \in B_{s-1}^{p, q}(\partial \Omega)
$$

is well-defined, linear and bounded, i.e.,

$$
\left\|\partial_{\nu}^{f} u\right\|_{B_{s-1}^{p, q}(\partial \Omega)} \leq C\left(\|f\|_{B_{s+\frac{1}{p}-2,0}^{p, q}(\Omega)}+\|u\|_{B_{s+\frac{1}{p}}^{p, q}(\Omega)}\right)
$$

holds, and which reduces to (2.3) when $p, q>1$. Furthermore, there exists a linear, bounded, right-inverse of (2.4).

Similar conclusions are valid in the context of Triebel-Lizorkin spaces, for the map

$$
\left\{(u, f) \in F_{s+\frac{1}{p}}^{p, q}(\Omega) \oplus F_{s+\frac{1}{p}-2,0}^{p, q}(\Omega): \Delta u=\left.f\right|_{\Omega}\right\} \ni(u, f) \mapsto \partial_{\nu}^{f} u \in B_{s-1}^{p, p}(\partial \Omega),
$$

with suitable values of $q$.

The above result has also pedagogical value since it anticipates the important role played by the scales $B_{s+\frac{1}{p}-2,0}^{p, q}(\Omega), F_{s+\frac{1}{p}-2,0}^{p, q}(\Omega)$ when dealing with the Green potential $\mathbf{G}_{N}$ later on.

Compared with the work in [9], [32], the principal new difficulty in the present setting is that, for $p<1$, duality techniques cannot easily be implemented. Instead, our proof relies on a different representation of $\partial_{\nu}$ which makes essential use of atomic decompositions of $B_{\alpha, 0}^{p, q}(\Omega)$ and $F_{\alpha, 0}^{p, q}(\Omega)$ with atoms individually supported in $\bar{\Omega}$; cf. [4] in the case of Hardy spaces.

\section{Poisson boundary value problems}

Consider the Poisson boundary value problem for the Laplacian in a domain $\Omega \subset \mathbb{R}^{n}$

$$
\Delta u=f \text { in } \Omega, \quad B u=g \text { on } \partial \Omega,
$$

with either Dirichlet $(B u:=\operatorname{Tr} u)$ or Neumann $\left(B u:=\partial_{\nu}^{f} u\right)$ boundary conditions.

For domains with smooth boundaries, the classical regularity theory of Agmon, Douglis and Nirenberg [3] has been extended in [21] to the scales of Besov and Triebel-Lizorkin spaces $B_{s}^{p, q}$ and $F_{s}^{p, q}$ with $0<p, q \leq \infty$ and $s \in \mathbb{R}$. In the case of domains with isolated singularities, a satisfactory theory has been developed in [16] for data in Besov spaces with $p>1$, and in [24] for suitable weighted Sobolev spaces.

The first systematic study of (3.1) with Dirichlet boundary conditions on Sobolev-Besov spaces on Lipschitz domains was undertaken by D. Jerison and C. Kenig [20] via harmonic measure techniques. Subsequently, their results have been reproved in [9] with an approach which relies on the method of boundary layer potentials. In particular, this allowed the authors to deal with Neumann 
boundary conditions as well; cf. also [32] for a different approach. However, both the techniques and the main results in [9], [20], [32], are largely limited to the case $p \geq 1$. Note that all Besov and Triebel-Lizorkin spaces with $\min \{p, q\}<1$ fail to be locally convex. Furthermore, below $p=1$, the nature of the trace operator changes as well.

The Theorems 3.1-3.2 below describe the most general range of indices for which the Poisson problem for the Laplacian equipped with Dirichlet or Neumann boundary conditions is well-posed on Besov and Triebel-Lizorkin spaces in Lipschitz domain.

Theorem 3.1. Let $\Omega$ be a bounded Lipschitz domain in $\mathbb{R}^{n}, n \geq 3$, and, for $\frac{n-1}{n}<p \leq \infty, 0<q \leq \infty,(n-1)\left(\frac{1}{p}-1\right)_{+}<s<1$, consider the following boundary value problem:

$$
\Delta u=f \in B_{s+\frac{1}{p}-2}^{p, q}(\Omega), \quad u \in B_{s+\frac{1}{p}}^{p, q}(\Omega), \quad \operatorname{Tr} u=g \in B_{s}^{p, q}(\partial \Omega) .
$$

Then there exists $\varepsilon=\varepsilon(\Omega) \in(0,1]$ such that (3.2) is well-posed if the pair $(s, p)$ satisfies one of the following four conditions:

$$
\begin{aligned}
(I): & \frac{n-1}{n-1+\varepsilon}<p \leq 1 \quad \text { and }(n-1)\left(\frac{1}{p}-1\right)+1-\varepsilon<s<1 \\
(I I): & 1 \leq p \leq \frac{2}{1+\varepsilon} \quad \text { and } \quad \frac{2}{p}-1-\varepsilon<s<1 \\
(I I I): & \frac{2}{1+\varepsilon} \leq p \leq \frac{2}{1-\varepsilon} \quad \text { and } 0<s<1 \\
(I V): & \frac{2}{1-\varepsilon} \leq p \leq \infty \text { and } 0<s<\frac{2}{p}+\varepsilon
\end{aligned}
$$

When $n=2$, then the same is true with the conditions $(I)-(I V)$ above replaced by

$$
\begin{aligned}
\left(I^{\prime}\right): & \frac{2}{1+\varepsilon} \leq p \leq \frac{2}{1-\varepsilon} \text { and } 0<s<1 \\
\left(I I^{\prime}\right): & \frac{2}{3+\varepsilon}<p<\frac{2}{1+\varepsilon} \text { and } \frac{1}{p}-\frac{1+\varepsilon}{2}<s<1 \\
\left(I I I^{\prime}\right): & \frac{2}{1-\varepsilon}<p \leq \infty \text { and } 0<s<\frac{1}{p}+\frac{1+\varepsilon}{2} .
\end{aligned}
$$

In the class of Lipschitz domains this result is sharp (for $\partial \Omega \in C^{1}$ then one may take $\varepsilon=1)$. Finally, analogous well-posedness results hold on the TriebelLizorkin scale.

It is illuminating to point out that the collection of all pairs $(s, 1 / p)$, such that the indices $s, p$ satisfy (3.3), can be identified with the two-dimensional hexagonal region depicted below: 


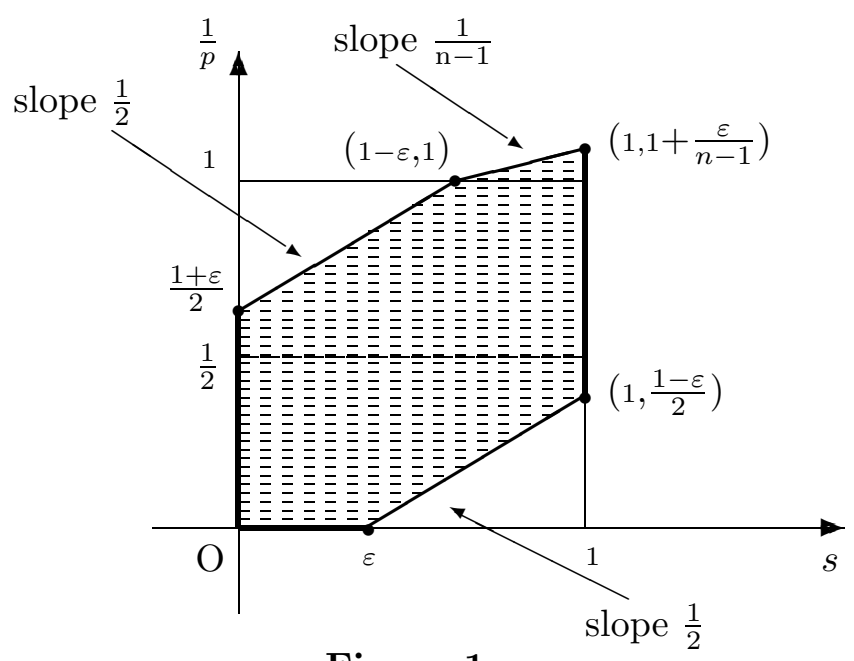

Figure 1.

An appropriate interpretation applies to the set of conditions $\left(I^{\prime}\right)-\left(I I I^{\prime}\right)$.

Regarding the Poisson problem with Neumann boundary conditions we have:

Theorem 3.2. Let $\Omega$ be a bounded, connected Lipschitz domain in $\mathbb{R}^{n}$ and, for $\frac{n-1}{n}<p \leq \infty, 0<q \leq \infty$, and $(n-1)\left(\frac{1}{p}-1\right)_{+}<s<1$, consider the following boundary value problem:

$$
\Delta u=\left.f\right|_{\Omega}, \quad f \in B_{s+\frac{1}{p}-2,0}^{p, q}(\Omega), \quad u \in B_{s+\frac{1}{p}}^{p, q}(\Omega), \quad \partial_{\nu}^{f} u=g \in B_{s-1}^{p, q}(\partial \Omega),
$$

where the data are assumed to satisfy the necessary compatibility condition

$$
\langle f, 1\rangle=\langle g, 1\rangle .
$$

Then there exists $\varepsilon=\varepsilon(\Omega) \in(0,1]$ such that (3.5) has a unique, modulo constants, solution if the pair $s, p$ satisfies either one of the four conditions in (3.3) when $n \geq 3$, or one of the three conditions in (3.4) when $n=2$. When $\partial \Omega \in C^{1}$, then one can take $\varepsilon=1$ in each case.

An analogous well-posedness result holds on the Triebel-Lizorkin scale.

Our strategy is to reduce matters to solving a homogeneous boundary problem for the Laplacian with Dirichlet and Neumann boundary conditions, respectively. Since the Newtonian potential in free space is smoothing of order two (a direct consequence of the Littlewood-Paley characterization of the spaces involved), the two key ingredients in this scenario are: (i) the trace/extension theory in $\S 2$, and (ii) the well-posedness of the appropriate homogeneous boundary problems. Our approach for dealing with the latter issue relies on singular integral operators. 


\section{Regularity of Green potentials in Lipschitz domains}

Recall from the introduction that $\mathbf{G}_{D}, \mathbf{G}_{N}$ are the Green operators for the Laplacian equipped with homogeneous Dirichlet and Neumann boundary conditions, respectively. In the case of the Neumann problem, it is understood that $u:=\mathbf{G}_{N} f$ solves $\Delta u=\left.f\right|_{\Omega}-|\Omega|^{-1}\langle f, 1\rangle$ in $\Omega, \partial_{\nu}^{f} u=0$ on $\partial \Omega$, and that $u$ is normalized so that $\langle u, 1\rangle=0$.

In this section we concentrate on the mapping properties of $\mathbf{G}_{D}, \mathbf{G}_{N}$ on Lipschitz domains. When $\Omega=\mathbb{R}^{n}$, or even when $\partial \Omega \in C^{\infty}$, both $\partial_{x_{j}} \partial_{x_{k}} \mathbf{G}_{D}$ and $\partial_{x_{j}} \partial_{x_{k}} \mathbf{G}_{N}, 1 \leq j, k \leq n$, fall under the scope of the classical theory of singular integral operators of Calderón-Zygmund type, but this fails to be the case in Lipschitz domains. The theorem below is the central result.

Theorem 4.1. For each bounded, connected Lipschitz domain $\Omega$ in $\mathbb{R}^{n}$ there exists $\varepsilon=\varepsilon(\Omega) \in(0,1]$ with the following significance. Assume that $\frac{n-1}{n}<p \leq$ $\infty,(n-1)\left(\frac{1}{p}-1\right)_{+}<s<1$, are such that either one of the four conditions in (3.3) is satisfied, if $n \geq 3$, or one of the three conditions in (3.4) is satisfied, if $n=2$. Then, with $\alpha:=s+\frac{1}{p}-2$, the operator

$$
\mathbf{G}_{D}: B_{\alpha}^{p, q}(\Omega) \longrightarrow B_{\alpha+2}^{p, q}(\Omega) \quad \text { if } 0<q \leq \infty
$$

is well-defined and bounded.

Similar results hold for the case of (homogeneous) Neumann boundary conditions. Specifically, retaining the same assumptions on the indices as before, the operator

$$
\mathbf{G}_{N}: B_{\alpha, 0}^{p, q}(\Omega) \longrightarrow B_{\alpha+2}^{p, q}(\Omega) \quad \text { if } 0<q \leq \infty
$$

is well-defined and bounded.

These results are sharp in the class of Lipschitz domains. For $\partial \Omega \in C^{1}$ one can take $\varepsilon=1$.

Analogous results hold on the Triebel-Lizorkin scale.

The two-dimensional hexagonal region consisting of all points with coordinates $(\alpha, 1 / p)$ such that $\alpha, p$ are as in the statement of Theorem 4.1 is shown below: 


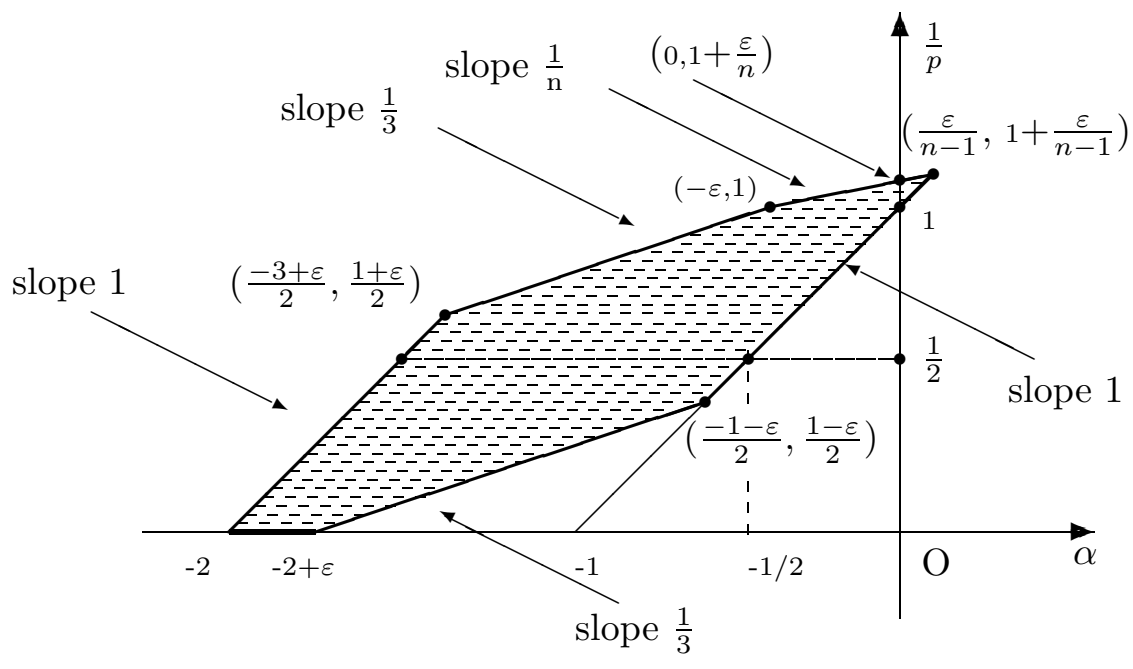

Figure 2

Theorem 4.1 is a direct consequence of Theorems 3.1-3.2, given the trace results from $\S 2$. Let $\nabla^{k}, k=0,1,2, \ldots$, stand for generic partial derivatives of order $k$.

Corollary 4.2. Retain the notation and hypotheses made in Theorem 4.1 on the indices involved and the domain $\Omega$. Then the operators

$$
\nabla^{2} \mathbf{G}_{D}: B_{\alpha}^{p, q}(\Omega) \longrightarrow B_{\alpha}^{p, q}(\Omega), \quad \nabla^{2} \mathbf{G}_{N}: B_{\alpha, 0}^{p, q}(\Omega) \longrightarrow B_{\alpha}^{p, q}(\Omega)
$$

are well-defined and bounded for each $0<q \leq \infty$.

Similar results hold on the Triebel-Lizorkin scale, in particular, for Hardy spaces. To be more precise, recall that as a well-known corollary of the classical Littlewood-Paley theory, the Triebel-Lizorkin space $F_{\alpha}^{p, q}\left(\mathbb{R}^{n}\right)$ reduces to the local Hardy class $h^{p}\left(\mathbb{R}^{n}\right.$ ) (cf. [15]) if $0<p<\infty, q=2, \alpha=0$ (as is well-known, the latter coincides with the Lebesgue class $L^{p}\left(\mathbb{R}^{n}\right)$ if $\left.1<p<\infty\right)$. Then, for $0<p<\infty$ and $\Omega \subset \mathbb{R}^{n}$ Lipschitz domain, we set $h^{p}(\Omega):=F_{0}^{p, 2}(\Omega)$ and $h_{0}^{p}(\Omega):=F_{0,0}^{p, 2}(\Omega)$ (so that $h^{p}(\Omega)=h_{0}^{p}(\Omega)=L^{p}(\Omega)$ if $\left.1<p<\infty\right)$. These Hardy spaces on domains have been previously introduced and studied in [4], [5], [6] and $[25]$.

Corollary 4.3. There exists $\varepsilon=\varepsilon(\Omega) \in(0,1]$ such that the operators

$$
\begin{aligned}
& \nabla^{2} \mathbf{G}_{D}: h^{p}(\Omega) \longrightarrow h^{p}(\Omega), \quad \text { whenever } \frac{n}{n+\varepsilon}<p<1, \\
& \nabla^{2} \mathbf{G}_{N}: h_{0}^{p}(\Omega) \longrightarrow h^{p}(\Omega), \quad \text { whenever } \frac{n}{n+\varepsilon}<p<1,
\end{aligned}
$$

are well-defined and bounded. Once again, one can take $\varepsilon=1$ whenever $\Omega$ has a $C^{1}$ boundary, in which case the range of $p$ 's in (4.4)-(4.5) becomes $\frac{n}{n+1}<p<1$. 
Corollary 4.3 is an extension of a result proved by D.-C. Chang, S.G. Krantz and E.M. Stein in [4] for domains $\Omega$ with a $C^{\infty}$ boundary. Their method relies on a reflection procedure across hyperplanes tangent to the boundary and, as such, makes essential use of the smoothness of $\partial \Omega$ (the differentiability properties of the reflection function closely mirror those of the boundary of the domain itself). Let us also mention that an alternative proof of (4.4) in $C^{\infty}$ domains, based on the Boutet de Monvel calculus of pseudodifferential operators, is in [21]; cf. the discussion on p. 32 loc. cit.

That one cannot allow $p \leq \frac{n}{n+1}$ even if the domain is smooth has already been observed in [4], [6]; see [6] for the appropriate function spaces which permit such an extension in smooth domains. The condition $p<1$ is also optimal. Indeed, in [20], D. Jerison and C. Kenig have constructed an example of a bounded domain $\Omega$ with $C^{1}$ boundary and a function $f \in C_{0}^{\infty}(\Omega)$ such that $\nabla^{2} \mathbf{G}_{D} f \notin L^{1}(\Omega)$, which sharpens a counterexample due to B. Dahlberg (cf. [7]). In particular, for this domain, $\nabla^{2} \mathbf{G}_{D}: h^{1}(\Omega) \longrightarrow h^{1}(\Omega)$ fails to be bounded and, hence, so does $\nabla^{2} \mathbf{G}_{D}: L^{p}(\Omega) \longrightarrow L^{p}(\Omega)$ for each $1<p<\infty$.

\section{Further applications}

5.1. The case of convex domains. According to an old result, proved by J. Kadlec in the 60's,

$$
\nabla^{2} \mathbf{G}_{D}: L^{2}(\Omega) \longrightarrow L^{2}(\Omega)
$$

is well-defined and bounded whenever $\Omega$ is a bounded convex domain in $\mathbb{R}^{n}$. The key to extending this result to the optimal range $1<p \leq 2$ is establishing a suitable end-point estimate for $p=1$. In the 90's, B. Dahlberg, G. Verchota and T. Wolff used (5.1) and elements of the Calderón-Zygmund theory to derive a weak-type estimate amounting to the boundedness of $\nabla^{2} \mathbf{G}_{D}: L^{1}(\Omega) \rightarrow$ $L^{1, \infty}(\Omega)$. See [1], [2] and [14], for a discussion. A related, stronger, result was proved by $\mathrm{V}$. Adolfsson in [1] where he established atomic estimates amounting to

$$
\nabla^{2} \mathbf{G}_{D}: h^{1}(\Omega) \longrightarrow L^{1}(\Omega),
$$

for a convex domain $\Omega$. His approach relied on the $L^{2}$ theory and the asymptotics at infinity for null-solutions of elliptic PDE's with $L^{\infty}$ coefficients due to J. Serrin and H. Weinberger (an idea pioneered by B. Dahlberg and C. Kenig in [8]).

The analogue of (5.1) in the case of Neumann boundary conditions has been known since the mid 70's (cf. [17], [16]), but optimal $L^{p}$ estimates have only been proved in the 90 's by V. Adolfsson and D. Jerison [2]. The key ingredient is showing that, for any bounded convex domain $\Omega$ in $\mathbb{R}^{n}$,

$$
\nabla^{2} \mathbf{G}_{N}: h_{0}^{1}(\Omega) \longrightarrow L^{1}(\Omega) .
$$

The Adolfsson-Jerison approach made, once again, essential use of the deep regularity theory for null-solutions of elliptic equations with bounded, measurable coefficients, alluded to above. As a result of our Hardy space estimate with 
$p<1$ in $\S 4$ we can give new, conceptually simple proofs to these key end-point estimates, and in fact sharpen them.

Theorem 5.1. Consider a bounded Lipschitz domain $\Omega \subset \mathbb{R}^{n}$ satisfying a uniform outer ball condition. Then

$$
\nabla^{2} \mathbf{G}_{D}: h^{1}(\Omega) \longrightarrow h^{1}(\Omega), \quad \nabla^{2} \mathbf{G}_{N}: h_{0}^{1}(\Omega) \longrightarrow h^{1}(\Omega)
$$

are bounded operators.

The idea is to simply interpolate between (4.4), (4.5), on the one hand, and the well-known $L^{2}$ theory on the other hand. Other weak-type results can be proved in a similar vein, such as $\nabla^{2} \mathbf{G}_{N}: L^{1}(\Omega) \longrightarrow L^{1, \infty}(\Omega)$.

5.2. Estimates for the gradient of Green potentials. The classical $L^{p}-L^{q}$ estimates for Green potentials in the half-space have been extended to Lipschitz domains by B. Dahlberg in [7], where he proved that

$$
\begin{aligned}
& \nabla \mathbf{G}_{D}: L^{p}(\Omega) \rightarrow L^{q}(\Omega), \quad 1<p<p_{n}+\varepsilon, \quad \frac{1}{q}=\frac{1}{p}-\frac{1}{n}, \\
& \nabla \mathbf{G}_{D}: L^{1}(\Omega) \rightarrow L^{\frac{n}{n-1}, \infty}(\Omega),
\end{aligned}
$$

where $\varepsilon=\varepsilon(\Omega)>0$ and $p_{n}:=\frac{3 n}{n+3}$ if $n \geq 3$ and $p_{n}:=\frac{4}{3}$ if $n=2$. Thus, informally, $\mathbf{G}_{D}$ behaves like a fractional integral operator of order one but only for a more restricted range of indices than what the classical Sobolev-HardyLittlewood theorem would normally warrant. In particular, if $G_{D}(x, y)$ denotes the Green function for $\Omega$, then, as opposed to the case when $\partial \Omega$ is smooth, the estimate $\left|\nabla_{x} G_{D}(x, y)\right| \leq C|x-y|^{-(n-1)}, x, y \in \Omega$, may fail for general Lipschitz domains.

A new proof of (5.5) was given in [19] based on a suitable functional analytic calculus for the Dirichlet Laplacian developed by the authors. Since both the Jerison-Kenig solution as well as Dahlberg's original argument ultimately depend on the maximum principle and on positivity, the results in these papers are restricted to the case of Dirichlet boundary conditions. The methods of the current paper allow for a uniform approach, which also yields stronger results. Recall that the Hardy space $h^{p}$ is isomorphic to $L^{p}$ whenever $p>1$. We have:

Theorem 5.2. Let $\Omega \subset \mathbb{R}^{n}$ be a bounded Lipschitz domain. There exists $\varepsilon=$ $\varepsilon(\Omega) \in(0,1]$ such that if $p(n, \varepsilon):=\left(\frac{n+3}{3 n}-\frac{\varepsilon}{3}\right)^{-1}$ for $n \geq 3$, and $p(n, \varepsilon)=\frac{4}{3-\varepsilon}$ for $n=2$, then for every $\frac{n}{n+\varepsilon}<p<p(n, \varepsilon)$ and $\frac{1}{q}=\frac{1}{p}-\frac{1}{n}$, the operators

$$
\nabla \mathbf{G}_{D}: h^{p}(\Omega) \longrightarrow L^{q}(\Omega), \quad \nabla \mathbf{G}_{N}: h_{0}^{p}(\Omega) \longrightarrow L^{q}(\Omega),
$$

are well-defined and bounded. In particular, (compare with (5.6))

$$
\begin{aligned}
& \nabla \mathbf{G}_{D}: h^{1}(\Omega) \rightarrow L^{\frac{n}{n-1}}(\Omega), \\
& \qquad \mathbf{G}_{N}: h_{0}^{1}(\Omega) \rightarrow L^{\frac{n}{n-1}}(\Omega), \\
& \quad \nabla \mathbf{G}_{N}: L^{1}(\Omega) \rightarrow L^{\frac{n}{n-1}, \infty}(\Omega),
\end{aligned}
$$


are bounded operators. The ranges of indices are optimal in the class of Lipschitz domains. When $\partial \Omega \in C^{1}$, one may take $\varepsilon=1$.

\section{References}

[1] V. Adolfsson, $L^{p}$-integrability of the second order derivatives of Green potentials in convex domains, Pacific J. Math., 159 (1993), 201-225.

[2] V. Adolfsson and D. Jerison, $L^{p}$-integrability of the second order derivatives for the Neumann problem in convex domains, Indiana Univ. Math. J., 43 (1994), 1123-1138.

[3] S. Agmon, A. Douglis and L. Nirenberg, Estimates near the boundary for solutions of elliptic partial differential equations satisfying general boundary conditions. I, Comm. Pure Appl. Math., 12 (1959), 623-727.

[4] D.-C. Chang, S.G. Krantz and E.M. Stein, $H^{p}$ theory on a smooth domain in $R^{N}$ and elliptic boundary value problems, J. Funct. Anal., 114 (1993), 286-347.

[5] D.-C. Chang, S.G. Krantz and E.M. Stein, Hardy spaces and elliptic boundary value problems, The Madison Symposium on Complex Analysis (Madison, WI, 1991), 119-131, Contemp. Math., 137 Amer. Math. Soc., Providence, RI, 1992.

[6] D.-C. Chang, G. Dafni and E.M. Stein, Hardy spaces, BMO, and boundary value problems for the Laplacian on a smooth domain in $\mathbb{R}^{n}$, Trans. Amer. Math. Soc., 351 (1999), 1605-1661.

[7] B.E.J. Dahlberg, $L^{q}$-estimates for Green potentials in Lipschitz domains, Math. Scand., 44 (1979), 149-170.

[8] B. Dahlberg and C. Kenig, Hardy spaces and the $L^{p}-$ Neumann problem for Laplace's equation in a Lipschitz domain, Ann. of Math., 125 (1987), 437-465.

[9] E. Fabes, O. Mendez and M. Mitrea, Boundary layers on Sobolev-Besov spaces and Poisson's equation for the Laplacian in Lipschitz domains, J. Funct. Anal., 159 (1998), 323-368.

[10] J.Franke and T.Runst, Regular elliptic boundary value problems in Besov-TriebelLizorkin spaces, Math. Nachr., 174 (1995), 113-149.

[11] M. Frazier and B. Jawerth, Decomposition of Besov spaces, Indiana Univ. Math. J., 34 (1985), 777-799.

[12] M. Frazier and B. Jawerth, The $\phi$-transform and applications to distribution spaces, Function spaces and applications, pp. 223-246 in Lecture Notes in Math., 1302, Springer, Berlin, 1988.

[13] M. Frazier and B. Jawerth, A discrete transform and decompositions of distribution spaces, J. Funct. Anal., 93 (1990), 34-170.

[14] S. Fromm, Potential space estimates for Green potentials in convex domains, Proc. Amer. Math. Soc., 119 (1993), 225-233.

[15] D. Goldberg, A local version of real Hardy spaces, Duke Math. J., 46 (1979), 27-42.

[16] P. Grisvard, Elliptic problems in nonsmooth domains, Monographs and Studies in Mathematics, 24 Pitman Boston, MA, 1985.

[17] P. Grisvard, G. Iooss, Problèmes aux limites unilatéraux dans des domaines non réguliers, Publications des Séminaires de Mathématiques, Université de Rennes, 9 (1975), $1-26$.

[18] B. Jawerth, The trace of Sobolev and Besov spaces if $0<p<1$, Studia Math., 62 (1978), 65-71.

[19] D. Jerison and C. Kenig, The functional calculus for the Laplacian on Lipschitz domains, Journés "Équations aux Dérivés Partielles," Exp. No. IV, 10 pp., École Polytech., Palaiseau, 1989.

[20] D. Jerison and C. Kenig, The inhomogeneous Dirichlet problem in Lipschitz domains, J. Funct. Anal., 130 (1995), 161-219.

[21] J. Johnsen, Elliptic boundary problems and the Boutet de Monvel calculus in Besov and Triebel-Lizorkin spaces, Math. Scand., 79 (1996), 25-85. 
[22] A. Jonsson and H. Wallin, Function spaces on subsets of $\mathbb{R}^{n}$, Math. Rep., 21984.

[23] C.E. Kenig, Harmonic analysis techniques for second order elliptic boundary value problems, CBMS Regional Conference Series in Mathematics, 83 AMS, Providence, RI, 1994.

[24] V.A. Kozlov, V.G. Maz'ya and J. Rossmann, Spectral problems associated with corner singularities of solutions to elliptic equations, Mathematical Surveys and Monographs, AMS 852001.

[25] A. Miyachi, $H^{p}$ spaces over open subsets of $R^{n}$, Studia Math., 95 (1990), 205-228.

[26] S.M. Nikol'skiı̌, Approximation of functions of several variables and imbedding theorems, $2^{\text {nd }}$ edition, Nauka, Moscow, 1977.

[27] J.Peetre, New thoughts on Besov spaces, Duke University Mathematics Series, 1 Durham, N.C., 1976.

[28] E.M.Stein, Singular integrals and differentiability properties of functions, Princeton Mathematical Series, No. 30 Princeton University Press, Princeton, N.J. 1970.

[29] R.S.Strichartz, The Hardy space $H^{1}$ on manifolds and submanifolds, Canad. J. Math., 24 (1972), 915-925.

[30] H. Triebel, Interpolation theory, function spaces, differential operators, VEB Deutscher Verlag der Wissenschaften, Berlin, 1978.

[31] H. Triebel, Theory of function spaces, Monographs in Mathematics, 78 Birkhäuser Verlag, Basel, 1983.

[32] D.Z. Zanger, The inhomogeneous Neumann problem in Lipschitz domains, Comm. Partial Differential Equations, 25 (2000), 1771-1808.

Department of Mathematics, University of Missouri at Columbia, Columbia, MO 65211, USA

E-mail address: svitlana@math.missouri.edu

E-mail address: marius@math.missouri.edu 